\title{
A New Generation of Los Alamos Opacity Tables
}

\author{
J. Colgan, D. P. Kilcrease, N. H. Magee, M. E. Sherrill, J. Abdallah, Jr., P. Hakel, C. J. Fontes, \\ J. A. Guzik, K. A. Mussack \\ Los Alamos National Laboratory, Los Alamos, NM 87545
}

\begin{abstract}
We present a new, publicly available, set of Los Alamos OPLIB opacity tables for the elements hydrogen through zinc. Our tables are computed using the Los Alamos ATOMIC opacity and plasma modeling code, and make use of atomic structure calculations that use fine-structure detail for all the elements considered. Our equation-of-state (EOS) model, known as ChemEOS, is based on the minimization of free energy in a chemical picture and appears to be a reasonable and robust approach to determining atomic state populations over a wide range of temperatures and densities. In this paper we discuss in detail the calculations that we have performed for the 30 elements considered, and present some comparisons of our monochromatic opacities with measurements and other opacity codes. We also use our new opacity tables in solar modeling calculations and compare and contrast such modeling with previous work.
\end{abstract}

Subject headings: opacities, atomic data, solar mixture

\section{Introduction}

The radiative opacity is a fundamental property of a material that determines the amount of radiation absorbed and scattered (Huebner \& Barfield 2014). In general, the opacity is dependent on the radiation temperature, material temperature and density of the material as well as the wavelength of the incoming radiation. The knowledge of material opacities are crucial in determining the transport of radiation through a material and therefore both quantities play a major role in stellar modeling including stellar evolution, pulsation, and in determining large-scale stellar quantities such as elemental abundance, temperature profiles, etc. Thermodynamic equilibrium is reached when the material and the radiation are at the same temperature and populations are in steady-state, a scenario often encountered in stars, and this allows the ready determination of material emissivity from the opacity via Kirchhoff's law. Stellar models have thus relied on tables of opacities computed in local thermodynamic equilibrium (LTE) for a range of elements present in the system of interest. Long-term intensive efforts to produce accurate and comprehensive opacity tables have been underway for many years, with notable efforts being the Opacity Project (OP) (Seaton et al. 1994; Seaton \& Badnell 2004; Badnell et al. 2005), the Lawrence Livermore National Laboratory OPAL opacity tables (Rogers \& Iglesias 1992; Iglesias and Rogers 1996), and 
more recently opacity tables produced using the OPAS code (Blancard et al. 2012). The OPAS code has very recently been used to compute opacities for solar mixtures (Mondet et al. 2015; Le Pennec et al. 2015), and improved agreement with helioseismic observations was reported. The SCO-RCG code (Pain et al. 2015) also appears to be a powerful method with which to compute opacities. At Los Alamos National Laboratory, tables of opacities have been computed using the LEDCOP code (Magee et al. 1995) in the 1990s and these OPLIB tables have successfully been used in solar modeling (Neuforge-Verheecke et al. 2001; Neuforge et al. 2001).

In recent years, it has become apparent that more refined opacity calculations could be useful in stellar modeling. In particular, the fairly recent discovery that the revision in the solar elemental abundances (Asplund et al. 2005) has destroyed the previously good agreement that existed between standard solar model predictions made using older solar elemental abundances (Grevesse \& Noels 1993) and helioseismic observations (Bahcall et al. 2005) has led to renewed scrutiny of the opacities used in such solar models. It was quickly noted (Serenelli et al. 2009) that an increase in opacity of some of the major solar elements of between 5-20\% in the solar radiative zone would restore the agreement between helioseismology and the solar models, although the two main sets of tables used in solar modeling (OP and OPAL) are in reasonably close agreement in this zone. Although even more recent studies have slightly revised the new solar abundances (Asplund et al. 2009), the 'solar abundance problem' is still not resolved (Guzik \& Mussack 2010). Opacities have also been postulated as the source of discrepancies between observations of pulsations of $\beta$-Cepheids and modeling, and in particular significant differences have been found between models when either OP or OPAL opacity tables are used (Daszyńska-Daszkiewicz \& Walczak 2009, 2010; Daszyńska-Daszkiewicz \& et al. 2013; Cugier 2012). More recent work by Walczak et al. (2015), which uses the new opacity tables described in this paper, produces improved agreement with observed pulsations for B stars.

These considerations, coupled with larger computational resources and more robust physical models, have led to a new opacity effort at Los Alamos National Laboratory (LANL) using the ATOMIC code (Magee et al. 2004; Hakel et al. 2006). The aim of this effort is to supplant the existing LANL OPLIB opacity tables previously computed using LEDCOP with a new generation of opacity tables computed using ATOMIC. This effort has been completed for all elements from hydrogen through zinc and our tables are available online 1 . The purpose of this paper is to describe in detail calculations that have been performed in generating these new opacity tables and to compare and contrast our new opacities with available measurements and previous theoretical work. Several previous publications (Colgan et al. 2013a, b, 2015) have described a few aspects of our ATOMIC calculations, including comparisons (Colgan et al. 2015) of the monochromatic opacity against several sets of opacity measurements made in the 1990s (Foster et al. 1988; Springer et al. 1992; Perry et al. 1991; Winhart et al. 1996). In this paper we also discuss comparisons of our opacity calculations against the opacity measurements made using the Sandia National Laboratory

\footnotetext{
${ }^{1}$ http://aphysics2.lanl.gov/opacity/lanl
} 
Z-pinch machine (Bailey et al. 2007, 2015). Finally, we also present new solar mixture opacity calculations and discuss the implications of the new opacities in solar modeling.

\section{Theoretical Methods}

Our opacity calculations were made using the ATOMIC code. ATOMIC is a multi-purpose plasma modeling code (Magee et al. 2004; Hakel et al. 2006; Fontes et al. 2015) that can be run in LTE or non-LTE mode. A major strength of ATOMIC is the ability to easily run at various levels of refinement (Fontes et al. 2016). For example, depending on the atomic datasets available, one can run with atomic data generated in the configuration-average approximation, or in fine-structure detail. The atomic data used in this work were generated with the semi-relativistic capability in the Los Alamos suite of atomic physics codes. An overview of this capability has recently been provided by Fontes et al. (2015).

The overall aim of our new opacity calculations is to compute a set of monochromatic opacities and Rosseland mean opacities for the elements hydrogen through zinc and for a wide range of temperatures and densities. We provide opacities over a temperature $(T)$ range of $0.5 \mathrm{eV}$ up to $100 \mathrm{keV}$, and for mass densities that span at least 12 orders of magnitude, starting at mass densities of around $10^{-8} \mathrm{~g} / \mathrm{cm}^{3}$ or lower (depending on the temperature and element under consideration). We also provide Planck mean opacities, although our focus is primarily on the Rosseland mean opacity since it is usually the quantity of main interest in most astrophysical applications. We define the Rosseland mean opacity as (Weiss et al. 2004)

$$
\frac{1}{\kappa_{\mathrm{ROSS}}}=\frac{\int_{0}^{\infty} \frac{1}{\kappa_{\nu}} n_{\nu}^{3} \frac{\partial B_{\nu}}{\partial T} d \nu}{\int_{0}^{\infty} \frac{\partial B_{\nu}}{\partial T} d \nu}
$$

where $\nu$ is the photon frequency, $B_{\nu}$ is the Planck function (Huebner \& Barfield 2014), $n_{\nu}$ is the frequency-dependent refractive index defined by Armstrong et al. (2014), and $\kappa_{\nu}$ is the frequencydependent opacity. Our opacities are given in $\mathrm{cm}^{2} /$ gram. $\kappa_{\nu}$ is composed of various contributions that can be summarized as

$$
\kappa_{\nu}=\kappa_{\mathrm{BB}}+\kappa_{\mathrm{BF}}+\kappa_{\mathrm{FF}}+\kappa_{\mathrm{SCAT}},
$$

i.e. the sum of bound-bound (BB), bound-free (BF), free-free (FF), and scattering (SCAT) contributions. The first three of these contributions include a factor due to stimulated emission. In the following subsections we discuss in detail the various calculations of each of these contributions as well as several related issues.

\subsection{Choice of configuration model for opacity calculations}

For a complete opacity calculation it is crucial to include sufficient numbers of states (configurations, levels, etc) so that the calculation is converged with respect to contributions to the 
total absorption. In the ATOMIC calculations presented here, we used a similar set of configurations that were used in the older LEDCOP calculations. These configurations are then split into fine-structure levels by one of several methods as discussed in the following subsections. The new sets of configurations were initially chosen based on considerations of what configurations would be likely to retain significant population for the (large) density and temperature range over which the tables run. A general rule of thumb was that all configurations that had an energy within about two times the ionization energy of the ground configuration were included. From this list of configurations, we also included configurations that had one-electron excitations from the valence sub-shell up to the $n l \equiv 10 \mathrm{~m}$ subshell. Finally, in order to obtain reasonably complete boundbound contributions over wide photon energy ranges, we also included one-electron dipole-allowed promotions from all subshells of this list of configurations. This final list of configurations was then used in the calculations of the EOS and opacity. Some specific examples follow.

For H-like ions, the list of configurations is straightforward, and includes all configurations from $1 s$ through $10 \mathrm{~m}$. For a case with many more electrons, such as neutral Sc (ground configuration: $[\mathrm{Ar}] 3 d^{1} 4 s^{2}$, where [Ar] means the electron configuration corresponding to the ground state of neutral Ar) we choose configurations of the type (where now the Ar core is omitted in this listing): $3 d^{1} 4 s^{2}$, $3 d^{1} 4 s^{1} n l, 3 d^{1} 4 p^{2}, 3 d^{1} 4 p^{1} n l, 3 d^{1} 4 d^{2}, 3 d^{1} 4 d^{1} n l, 3 d^{1} 4 f^{2}, 3 d^{1} 4 f^{1} n l, 3 d^{3}, 3 d^{2} n l, 4 s^{2} n l, 4 s^{1} 4 p^{2}, 4 s^{1} 4 p^{1} n l$, $4 p^{3}, 4 p^{2} n l$. Again, $n l$ extends up to $10 \mathrm{~m}$. Contributions from levels with $n>10$ are included in an approximate manner (see following sections). This choice of configurations encompasses 384 configurations, and these are then used to promote an electron from each sub-shell to any (open) sub-shell that is available via a dipole promotion (i.e. where the orbital angular momentum of the jumping electron changes by \pm 1 ). For neutral Sc, this choice leads to a list of 21,144 configurations. Frequently, we find lists of configurations that are significantly larger than this, especially for ions with (near) half-filled shells in their ground configuration. For example, the total number of configurations employed for the Fe opacity table was more than 600,000 (for all ion stages of Fe).

We note that this prescription for choosing configurations does not guarantee that all possible configurations that may significantly contribute to an opacity are included. However, we believe that our approach is sufficiently inclusive that it is likely that more configurations are retained than needed for any given temperature and density, although this is difficult to explicitly demonstrate for every temperature/density point considered without even larger calculations. In Section 3 we do discuss the effect of increasing the number of configurations for one set of opacity calculations for Fe. We do note that the relative ease of choosing sets of configurations, and the guarantee of consistency between and within datasets, make this approach to choosing configurations viable for the generation of large-scale opacity tables.

\subsection{Bound-bound opacity contribution}

In the opacity calculations presented here, we make use of atomic data generated in a variety of ways. All atomic structure calculations were performed using the Los Alamos CATS code 
(Abdallah et al. 1988), a modern version of Cowan's codes (Cowan 1981). The calculations were carried out using the semi-relativistic Hartree-Fock, or HFR, option. For the Li-like, He-like and $\mathrm{H}$-like ions of all elements discussed here, we use atomic data generated at a fine-structure level of detail including full configuration-interaction between all configurations. For ion stages with more electrons than Li-like, inclusion of full configuration-interaction proved too computationally expensive. Instead, we employed a 'single-configuration' approximation, in which we include mixing among the pure $L S J$ basis states that arise from a given configuration (also known as intermediatecoupling; see Fontes et al. (2015) for details). Oscillator strengths are computed from wavefunctions containing the same limited amount of mixing. These calculations are accomplished in CATS by looping over transitions occurring between pairs of dipole-allowed configurations, which automatically exclude matrix elements between pure $L S J$ basis states that would arise from configurationinteraction. This option, by omitting the mixing between different configurations, greatly reduces the run time for large structure calculations, while retaining the total number of $L S J$ levels in the calculation. To improve the accuracy of $\Delta n=0$ transitions for $L$-shell ions, we perform a small configuration-interaction calculation for all possible $1 s^{2}[2]^{w}$ configurations, where $[2]^{w}$ represents all possible permutations of $w$ electrons in the $n=2$ shell. The resulting energies replace the (less accurate) energies for those levels computed within the single-configuration calculation, resulting in improved $L$-shell line positions in the frequency-dependent opacity. The above procedure results in a comprehensive set of level-resolved structure and oscillator strength data and was implemented for the Be-like through neutral stages of the elements Be through Si.

For elements beyond Si (i.e. P through Zn) we used a mixed-UTA (MUTA) approach (Mazevet \& Abdallah 2006) for all ions from Be-like through the neutral stage. The MUTA method retains all of the strongest fine-structure lines in a given transition array, which allows an accurate spectral description to be generated from a set of configuration-average populations. This approach allows single-configuration fine-structure detail to be included in a relatively inexpensive computational manner. The generation of atomic data for a full level-resolved calculation is too computationally intensive at present. In the calculations presented here, we retained all fine-structure lines for transition arrays that contained less than $10^{5}$ lines within the array. We have found that, for almost all conditions of interest, this parameter choice allows essentially all lines of importance to be explicitly included in the bound-bound opacity contribution. A histogram approach that was introduced by Abdallah et al. (2007) to speed up computation of bound-bound contributions to spectra was also modified to work with the MUTA approach and was found to significantly speed up large-scale calculations with essentially no loss in accuracy. The histogram approach was used for the computation of opacity tables for all elements apart from $\mathrm{H}$, which did not have significant run-times and so did not require this speed up option. The MUTA approach has been compared to previous Fe transmission measurements, where excellent agreement was obtained (Bailey et al. 2007) for a temperature of $160 \mathrm{eV}$ and an electron density of a few times $10^{21} \mathrm{~cm}^{-3}$. Testing shows that our use of the MUTA approximation appears to be accurate for temperatures above around $10 \mathrm{eV}$. Below this temperature, the assumption of statistical splitting of the population of a configuration into its constituent levels may not be as accurate. This is, in part, due to the increasing importance of the 
Boltzmann factor $e^{-\Delta E / k T}$ at lower temperatures, which appears in the expression for calculating a given population. Using the configuration-average transition energy, $\Delta E_{C A}$, may not be a good approximation for the associated fine-structure transition energies for some types of transitions.

\subsection{Bound-free opacity contribution}

The bound-free contribution to the opacities presented here are computed using configurationaverage distorted-wave photoionization cross sections calculated using the GIPPER ionization code (Clark et al. 1991). We include photoionization contributions between all possible configurations in neighboring ion stages. We also include bound-free contributions from the photodetachment of $\mathrm{H}^{-}$using the data provided by Geltman (1962). The occupation probabilities that are discussed in Section 2.7 are used to model the merging of a Rydberg series with its corresponding boundfree edge (Hubeny et al. 1994; Däppen 1987). This 'edge blending' approach results in smoother monochromatic opacities near a bound-free edge by accounting for the redistribution of population from bound to continuum states via pressure ionization, while still conserving oscillator strength. The edge blending procedure fills the gap between the highest included $n$-value and the original edge, and is performed down to the lowest $n$ value of the Rydberg series under consideration.

\subsection{Free-free opacity contribution}

The free-free contribution to the opacity computed by ATOMIC (also known as inverse Bremsstrahlung) is computed using the tables provided by Nakagawa et al. (1987), results that are correct for any plasma degeneracy, and corrected for plasma screening based on methods developed by Green (1958, 1960); Armstrong et al. (2014). This contribution is also corrected at low frequencies by incorporating effects due to multiple electron-ion collisions (Iglesias 2010). We also include freefree contributions from the $\mathrm{H}^{-}$(Geltman 1965), $\mathrm{He}^{-}$(Somerville 1965) and $\mathrm{C}^{-}$ions (Bell et al. 1988). These latter contributions are important only at low temperatures below $1.0 \mathrm{eV}$ or so. We note that the inclusion of the free-free contribution used in ATOMIC is very similar to that in LEDCOP, apart from our use of the multiple electron-ion collision correction at low frequencies. The $\mathrm{C}^{-}$free-free contribution was also not included in the LEDCOP calculations. A recent study by Armstrong et al. (2014) used an ab-initio partial wave expansion (and more computationally intensive) approach to compute the free-free contribution. It was found that similar results were obtained compared to the use of the tables of Nakagawa et al. (1987) for most conditions of interest.

\subsection{Photon scattering opacity contribution}

For photon scattering from free electrons, we use the early tables of Sampson (Sampson 1959) for Compton scattering, modified to include the effects of Pauli blocking and collective effects, 
and also include plasma non-ideality effects (Kilcrease \& Magee 2001) due to strong coupling and electron degeneracy. The use of Compton scattering lowers the opacity at high photon energies as compared to the use of Thomson scattering. The inclusion of Pauli blocking and collective effects lead to a decrease in the Rosseland mean opacity at very high densities. We also note that, when computing the number of free electrons for Compton scattering, we consider bound electrons as free when the photon energy is equal to or greater than the electron's binding energy. Finally, we also include the effects of Rayleigh scattering for the low energy side of the lowest energy transition of the ground state of all neutral atoms. The formulae used for this contribution for various neutral species are given in the Appendix. We note that at low temperatures, where neutral species contributions often dominate the opacity, the Rayleigh scattering contribution can be very important.

\subsection{Line broadening}

We now discuss the line broadening packages used in ATOMIC. As is well known, Stark broadening of H-like and He-like lines are important in the opacity from such ions, and this was included following the procedure of Lee (1988). This procedure was modified within ATOMIC to make use of atomic data computed from our atomic structure calculations (i.e. CATS (Abdallah et al. 1988)), rather than from data tables as originally proposed by Lee (1988). To make our calculations completely consistent, we included natural broadening within the Stark broadening package, and extended the temperature and density ranges over which Stark broadening is included. We also introduced neutral resonance and neutral van der Waals broadening, that is line broadening contributions due to the presence of other neutral atoms within the plasma through van der Waals interactions (Hindmarsh et al. 1967; Schwerdtfeger 2006) and through resonance effects (Ali et al. 1965, 1966), into our line broadening package. These processes were found to give a small contribution in low temperature regions, where neutral species dominate. It was especially relevant for He, where the large ionization potential of the neutral atom results in a fairly wide temperature range over which neutral contributions to the opacity are important. For lines not treated by the Stark package we use a Voigt profile incorporating Doppler, natural, and, where applicable, neutral broadening. Electron collisional broadening is included using the approach of Armstrong (Armstrong et al. 1966).

A detailed study was also made of line shapes far from the line center. It was found that, again particularly for He, for cold temperatures the line-wing of the nearest bound-bound transition continues to dominate the opacity, even $10^{6}$ half-widths away from the line center. The question then arises as to what is the correct form of the line shape in such a region. Previous studies by Seaton et al. (1994) and Griem (1974) reached differing conclusions as to the form of this line shape. After some further consideration and based on the arguments of Heitler (2010), the choice was made that in the far red line wing of all absorption lines, the $\omega^{4}$ behavior used by Seaton et al. (1994) in the OP data was the most suitable, where $\omega$ is the photon angular frequency. This decision was 
based on consideration of the scattering and absorption processes within a QED framework. We note that this line shape feature can make a very large difference to the opacity in certain regions. For example, if the $\omega^{4}$ line shape is not used, the Rosseland mean opacity for He at low $(\sim 1$ $\mathrm{eV}$ ) temperatures, may increase by more than three orders of magnitude, since the far line wing of the nearest bound-bound transition is (essentially) the only contributor to the opacity at these conditions. At larger temperatures, and for other elements with smaller ionization potentials, this effect is much smaller.

\subsection{Equation-of-state used in opacity calculations}

The equation-of-state (EOS) model used in ATOMIC is known as ChemEOS (Hakel \& Kilcrease 2004; Kilcrease et al. 2015), which we summarize here. This approach is based on the minimization of the Helmholtz free energy in the chemical picture. Adopting this approach allows us to write the total free energy as

$$
F=F_{1}+F_{2}+F_{3}+F_{4}+F_{5}
$$

where in this equation $F_{1}$ represents the ideal gas of atoms and ions, $F_{2}$ represents the contribution associated with the internal energy of the atoms and ions, and $F_{3}$ represents the ideal Fermi electron gas free energy term. The internal energy $F_{2}$ term, given by

$$
F_{2}=\sum_{s \neq e} N_{s}\left(E_{s 1}-k T \ln \tilde{Z}_{s}\right)
$$

depends on the converged partition function defined as

$$
\tilde{Z}_{s}=\sum_{j} w_{s j} \exp \left(-\frac{E_{s j}-E_{s 1}}{k T}\right),
$$

where in these equations $N_{s}$ is the number of particles of species $s$ (not including electrons), $k T$ is the temperature, and $E_{s j}$ is the $j$ th-state energy. The convergence of the partition function is ensured through the occupation probabilities $w_{s j}$ that smoothly truncate the summation by progressively reducing the effective statistical weights of the excited states due to their perturbation by plasma effects. They are given by $w_{s j}=w_{s j}^{H S} Q_{s}\left(\beta_{s j}\right)$, where $w_{s j}^{H S}$ is a first-order hard-sphere contribution based on the size of the bound state (Hummer \& Mihalas 1988), $Q_{s}$ is the cumulative microfield distribution function with $\beta_{s j}$ the critical microfield (Potekhin et al. 2002). For $\mathrm{H}$ and He we use a screened microfield distribution (Potekhin et al. 2002), but for all other elements we use an unscreened microfield distribution due to the prohibitive cost of the screened distribution computation for systems with large numbers of states.

The hard-sphere occupation probability term $w_{s j}^{H S}$ is included for all atoms and ions, and results in the elimination of the (unphysical) atomic state populations at high densities. We also note that our partition function is extended from the $n=10$ principal quantum number value 
explicitly included in our list of configurations up to $n=100$ via analytic quantum defect terms. This extension was also performed in the previous LEDCOP calculations.

The $F_{4}$ term in Eq. (1) contains the Coulomb contributions (Chabrier \& Potekhin 1998; Potekhin \& Chabrier 2000) to the free energy and is broken up into three contributions: ionion, ion-electron, and electron-electron contributions. The ion-electron term within $F_{4}$ was recently modified in Kilcrease et al. (2015) to ensure that the effect of electron-ion binding is more consistently taken into account. The Coulomb interaction terms of Chabrier \& Potekhin (1998); Potekhin \& Chabrier (2000) include electron degeneracy to all orders and our modification also takes this electron degeneracy into account. The final term in Eq. (1), $F_{5}$, accounts for the finite size of the atom or ion through an excluded volume effect using an all-order hard sphere packing term. Detailed discussion of all these contributions can be found in Hakel \& Kilcrease (2004).

\subsection{The Los Alamos National Laboratory Opacity Website}

For over a decade, the Los Alamos OPLIB opacity tables have been accessible via a website: http://aphysics2.lanl.gov/opacity/lanl. This webpage has been updated recently to include access to the new OPLIB opacity tables that have been computed using the ATOMIC code. On the webpage, the user may request opacities for single elements or mixtures of elements (any arbitrary mixture of the elements hydrogen through zinc may be specified). The user may obtain monochromatic opacities (these include opacities on a temperature-scaled $u=h \nu / k T$ grid of 14,900 photon energies that are chosen to encompass a large photon energy range, and to provide a sufficient density of points in the region where the Rosseland weighting function is peaked), multigroup opacities, or Rosseland mean and Planck mean opacities. The total monochromatic opacities are tabulated and we also tabulate separately the absorption and scattering contributions, with the absorption contribution consisting of the first three terms of the right-hand-side of Eq. (2) and the scattering contribution as discussed in Section 2.5. We note that the Rosseland mean opacities available from our website are computed assuming a refractive index $n_{\nu}$ set equal to 1.0 above the plasma frequency, and to zero below that frequency, in the integration that appears in Eq. (1). This choice is made so that mixtures of opacities may be computed in a more straightforward manner. Mixtures of opacities are generated by mixing the pure-element OPLIB tables under the assumption of electron-temperature and electron-degeneracy equilibrium (Huebner \& Barfield 2014).

The user may choose to obtain opacities from the latest OPLIB tables (generated using ATOMIC) or from the previous set of OPLIB tables (generated using LEDCOP). The new ATOMICgenerated OPLIB tables are available on a more refined temperature grid, with 24 more isotherms available compared to the grid on which the previous LEDCOP calculations were made. This should help reduce interpolation errors that may arise when interpolating our opacity tables onto

a different temperature grid. Such issues were discussed in detail by Neuforge-Verheecke et al. (2001). Also, the new opacity tables generally extend to higher mass densities than were available from the OPLIB tables computed using LEDCOP. Finally, as a service to the community, opacity 
tables are available for a variety of pre-calculated mixtures of elements.

\section{Results}

\subsection{Single-Element Opacity Comparisons}

In our previous publications we have performed several sets of comparisons of our new opacity calculations with measurement and other theoretical opacity efforts that are available in the literature. For example, in Colgan et al. (2015) we compared monochromatic opacities of Al and Fe with various measurements performed in the 1990s (Foster et al. 1988; Springer et al. 1992; Perry et al. 1991; Winhart et al. 1996). Overall, good agreement was found with these measurements. We have also compared Rosseland mean opacities for the elements $\mathrm{H}$ and He (Colgan et al. 2013a), and $\mathrm{C}$ and $\mathrm{O}$ (Colgan et al. 2013b) with OP and OPAL data. Again, overall reasonable, although not perfect, agreement was found between the ATOMIC calculations and other opacity efforts. In Colgan et al. (2015) we also examined the opacity of transition metal elements at the somewhat lower temperature of $15.3 \mathrm{eV}$. We identified significant differences between our ATOMIC opacities and those generated using OP (Badnell et al. 2005) calculations for $\mathrm{Fe}, \mathrm{Ni}$, and $\mathrm{Cr}$. In particular, the systematic trend in the monochromatic opacity that is apparent in the ATOMIC calculations as one moves from $\mathrm{Cr}$ to $\mathrm{Fe}$ to $\mathrm{Ni}$ is not observed in the $\mathrm{OP}$ calculations. The differences in the monochromatic opacities of these elements lead to an increase of around a factor of two in the ATOMIC calculations of the Rosseland mean opacity compared to the OP calculations.

In figure 1 we examine the opacity of $\mathrm{Mg}$ at a temperature and density relevant to the base of the convection zone of the Sun, that is a temperature of $192.91 \mathrm{eV}$ and an electron density of $10^{23} \mathrm{~cm}^{-3}$. This monochromatic opacity was also examined by the OPAS team (Blancard et al. 2012). We compare the ATOMIC calculation to the OP calculations (available online at http://cdsweb.u-strasbg.fr/topbase) and find reasonable agreement in the broad features of the monochromatic opacity. However, we do note that the strong $\mathrm{He}_{\alpha}$ line at a photon energy of $1352 \mathrm{eV}$ is broader in the $\mathrm{OP}$ calculation than in the ATOMIC calculation. In the ATOMIC calculation, the Stark broadening of this line is computed using the package of Lee (Lee 1988), modified to utilize the atomic data available from CATS. It is unclear as to how the broadening of the He-like lines is computed within the OP calculations.

The broader $\mathrm{He}_{\alpha}$ line from the OP calculation was also noted by the work of Blancard et al. (2012), and the OPAS opacity appears quite close to the ATOMIC opacity shown in figure 1 . The inset of figure 1 shows the ionization balance of $\mathrm{Mg}$ for these conditions. Very close agreement is found between the OP, OPAS and ATOMIC calculations.

In figure 2 we examine the opacity of Fe and compare our ATOMIC calculations to the measurements of Bailey et al. (2007) made using the Sandia National Laboratory Z-pinch platform. We find very good agreement between our calculations and the 2007 measurements. There is ex- 


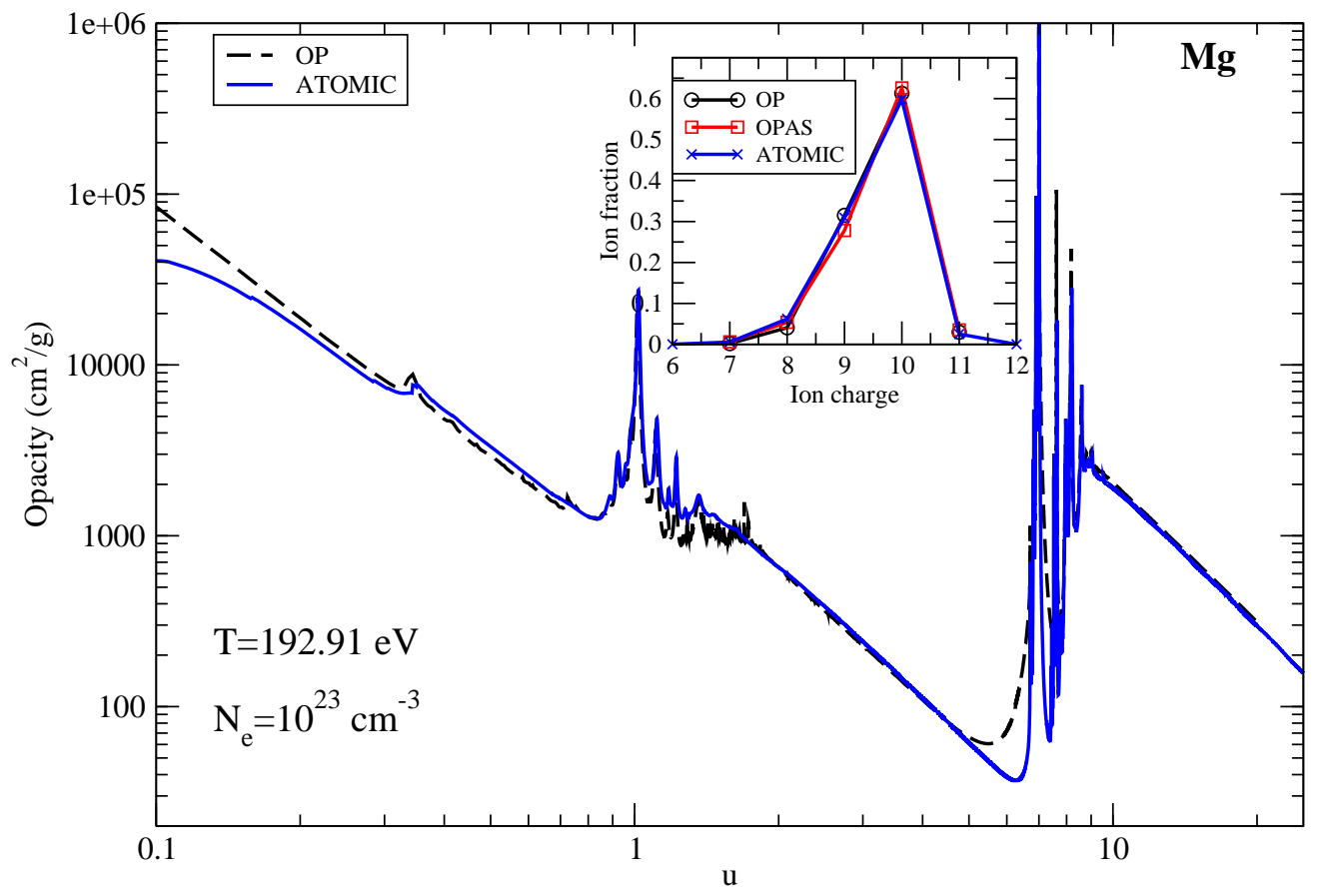

Fig. 1. - Comparison of Mg opacity (as a function of $u=h \nu / k T$ ) at a temperature of $192.91 \mathrm{eV}$ and an electron density of $10^{23} \mathrm{~cm}^{-3}$. The current ATOMIC calculation (blue curve) is compared with an OP calculation (black dashed line) (Badnell et al. 2005). The inset shows the ionization balance of $\mathrm{Mg}$ at the same conditions where we compare the current ATOMIC calculations (blue crosses) with OP (black circles) (Badnell et al. 2005) and OPAS (red squares) calculations (Blancard et al. 2012). 
cellent agreement between the measured and calculated line positions and valleys between the lines (which are important in Rosseland mean opacity calculations), as well as in the underlying opacity, although some difference is observed in some of the line heights. This may be due to an incomplete treatment of the effects of instrument resolution in the calculations. We note that similar calculations to the ones shown here were reported by Bailey et al. (2007) and were also made using the MUTA option in ATOMIC, but with a different atomic data set. The calculations shown in figure 2 were made using the same atomic models that were used to construct the Fe OPLIB table that is available through our opacity website.

We now discuss the more recent experiments that measured the opacity of Fe using the refurbished Sandia Z-pinch platform (Bailey et al. 2015), which produces more energy per shot. This, coupled with a change in the design of the tampers of the $\mathrm{Fe} / \mathrm{Mg}$ targets, enabled hotter temperatures and higher densities to be explored. (The Fe target is combined with layers of $\mathrm{Mg}$ so that the $\mathrm{Mg}$ lines can be used as a diagnostic to obtain estimates of the plasma electron density and temperature). The inferred conditions (from the $\mathrm{Mg}$ line diagnostics) implied a plasma temperature of $182 \pm 7 \mathrm{eV}$ with an electron density of $3.1 \pm 0.78 \times 10^{22} \mathrm{~cm}^{-3}$. However, ATOMIC calculations at those conditions (as shown in figure 3 of Bailey et al. (2015)) are in poor agreement with the opacity inferred from the experiment. In particular, although ATOMIC is in good agreement with the positions of the major line features, a persistent background discrepancy is found between measurement and calculation. We note especially the disagreement at the lowest wavelengths, where the bound-free contribution (from the $L$-shell) to the opacity dominates. As pointed out in Bailey et al. (2015), these measurements (and disagreements with theory), if confirmed, have important implications for solar opacities near the base of the radiative convection zone.

The large disagreement between the ATOMIC calculations and the Sandia measurements prompted us to re-examine many aspects of our calculations. In particular, the higher electron density inferred in the more recent measurements led to speculation (Bailey et al. 2015) that much of the population of the relevant Fe ion stages resided in excited states, and that this population may not be accurately portrayed in the calculations. To test this hypothesis, we constructed atomic data sets comprised of larger numbers of multiply-excited configurations than used in our normal Fe opacity tables (which already included a considerable number of configurations that represented multiply-excited-states). This was accomplished by including promotions of four electrons from the $L$-shell of the relevant ions that were populated in this calculation (which ranged from N-like through Mg-like Fe). The original ATOMIC calculations already included promotions of at least two electrons from the $L$-shell. This inclusion resulted in calculations that included around one order of magnitude more configurations than in the calculations used originally. This increased number of configurations was then used in the atomic structure calculations, and ATOMIC was again used to compute the resulting opacity. This effort led to only small changes in the monochromatic opacity compared to the original ATOMIC calculations, and in particular the bound-free opacity (which dominates the total opacity at lower wavelengths) was almost unchanged. We also examined several other aspects of our calculations. We tested the effects of inclusion of full configuration-interaction 


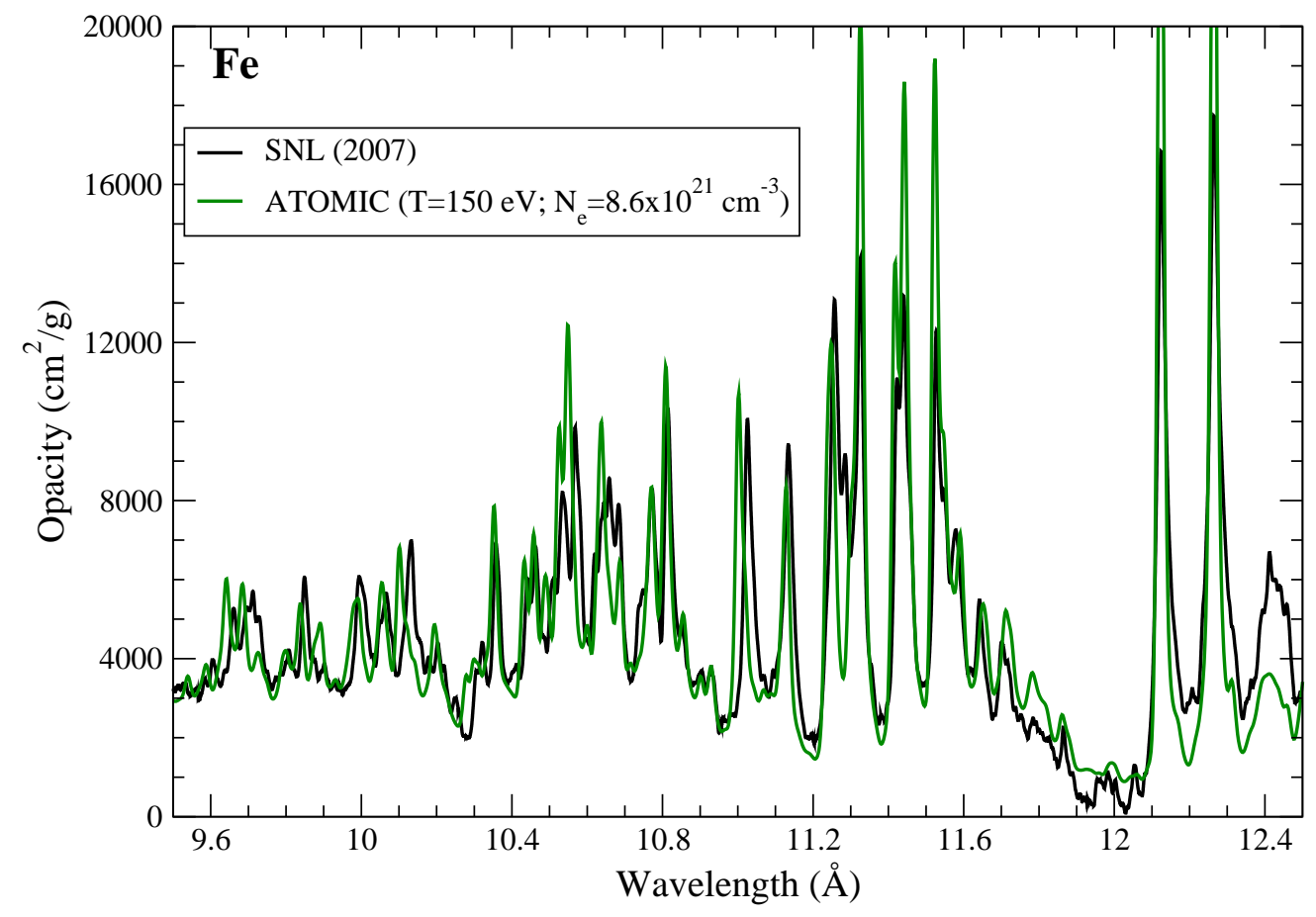

Fig. 2.- Comparison of Fe opacity measured in 2007 (Bailey et al. 2007) using the Sandia National Laboratory Z-pinch (black line) with an ATOMIC calculation at a temperature $(T)$ of $150 \mathrm{eV}$ and an electron density $\left(N_{e}\right)$ of $8.6 \times 10^{21} \mathrm{~cm}^{-3}$. The experimental conditions were inferred to be $T=156 \pm 6 \mathrm{eV}$ and $N_{e}=6.9 \times 10^{21} \mathrm{~cm}^{-3} \pm 25 \%$ (Bailey et al. 2007). 
(CI) within our models by constructing a smaller set of configurations for the relevant Fe ion stages for which a full CI calculation was feasible. The effects of full CI were found to be minor and again had almost no change on the resulting bound-free cross sections. We also tested the sensitivity of our calculations to the choice of screening microfield distribution in the occupation probability (as discussed in the previous section). Again, at these conditions, our calculations were not sensitive to the choice of screening within the microfield.

We conclude this discussion by noting that other modern opacity calculations also disagree with the Sandia measurements, as discussed in Bailey et al. (2015). We also note a recent paper (Iglesias 2015a) that implies that the Sandia measurements, if correct, are in apparent violation of oscillator strength sum rules. Another important related issue is related to the bound-free opacity of the Fe plasma at these conditions. If this is indeed underestimated in the calculations, this has serious implications for long-established methods in atomic physics for computing photoionization cross sections from highly-charged ions. Our approach to photoionization uses a distorted-wave approach, which is usually thought to be of acceptable accuracy for photoionization calculations from moderately- and highly-charged systems. On the other hand, the measurements reported in Bailey et al. (2015) have been subject to considerable scrutiny (Nagayama et al. 2014) in the search for experimental issues that might affect the measured opacity. No significant systematic errors that could artificially increase the measured opacity were reported by Nagayama et al. (2014). We look forward to independent verification of these measurements and to a resolution of this discrepancy.

We now turn to a discussion of opacities at conditions relevant to B stars. The pulsation properties of such stars were recently explored (Walczak et al. 2015) using our new Los Alamos opacities. Previous work (Gilles et al. 2011; Turck-Chièze et al. 2013) has cast some doubt on the use of the OP database for such systems. In particular, the Ni OP opacities, which made use of scaled atomic data (Badnell et al. 2005) were found to be in significant disagreement with several other sets of opacity calculations (Turck-Chièze et al. 2013). Studies of Fe opacities at these conditions, including previous ATOMIC calculations (Colgan et al. 2013a), also indicated that several important inner-shell transitions may have been omitted from the OP calculations, a conclusion also reached by Iglesias (2015b).

Since the differences between the OP database and more recent calculations for $\mathrm{Fe}$ and $\mathrm{Ni}$ have been documented, we here examine the opacity of $\mathrm{Cr}$ at conditions relevant for B stars. Figure 3 shows a comparison of the OP calculations and ATOMIC calculations of Cr at two sets of conditions. The upper panel shows the opacity at a temperature corresponding to $\log (T)=5.25$ (temperature $T$ in $\mathrm{K}$ ) and an electron density of $3.16 \times 10^{17} \mathrm{~cm}^{-3}$; this corresponds to conditions near the opacity bump ( $Z$-bump) that is evident when the Rosseland mean opacity is plotted as a function of temperature for constant $\log R$ values (where $R=\frac{\rho}{T_{6}^{3}}$, with $\rho$ the mass density in $\mathrm{g} / \mathrm{cm}^{3}$ and $T_{6}=10^{-6} T$ ). We find that both the OP and ATOMIC calculations produce monochromatic opacities that exhibit a dense forest of lines due to the very large number of bound-bound transitions that contribute to the total opacity at these conditions. Although it is difficult to make a meaningful comparison of the two calculations for such a dense spectrum, it does appear that the ATOMIC 

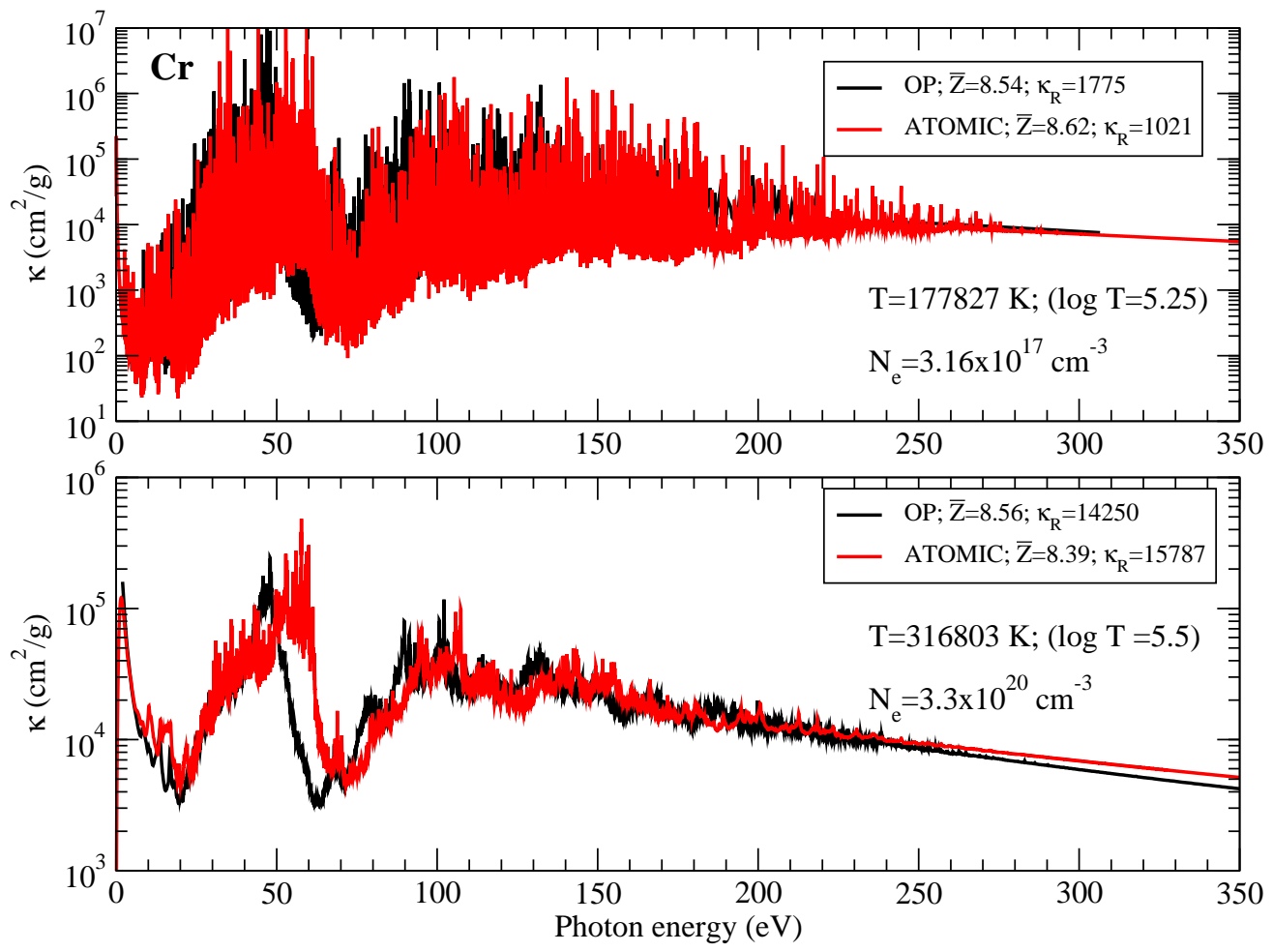

Fig. 3.- Comparison of the opacity of Cr from the OP database (Badnell et al. 2005) (black lines) and the current ATOMIC calculations (red lines) at two different sets of temperatures $(T)$ and electron densities $\left(N_{e}\right)$ as indicated. The average ionization $(\bar{Z})$, and Rosseland mean opacity $\left(\kappa_{\mathrm{R}}\right)$, in $\mathrm{cm}^{2} / \mathrm{g}$, are indicated in the figure captions. 
opacity is somewhat shifted to higher photon energies compared to the OP opacity. Furthermore, we note that the Rosseland mean opacity from the OP calculation is considerably higher than that from the ATOMIC calculation. In an effort to explore this further, in the lower panel of figure 3 we examine the Cr opacity at a higher temperature and electron density, following a strategy proposed by Turck-Chièze et al. (2013). This set of conditions was chosen to produce a similar ionization balance to the conditions in the upper panel. This is reflected by the reasonably similar average ionization $(\bar{Z})$ found for these conditions as indicated in the figure. The monochromatic opacity in the lower panel has features that are much broader than the narrow lines evident in the upper panel due to the larger electron density in this case, which causes the bound-bound features to broaden and merge with each other. Although for the conditions in the lower panel we find that the Rosseland mean opacities from OP and ATOMIC are closer than in the upper panel, we note that the ATOMIC calculations are again shifted to higher photon energies compared to the OP calculations. Since we remember that the OP calculation for $\mathrm{Cr}$ (as well as for $\mathrm{Ni}$ and $\mathrm{Mn}$ ) used scaled atomic data (Badnell et al. 2005), this shift may be due to this approximation. This is partially confirmed by comparison to LEDCOP calculations (not shown), which are in very good agreement with the ATOMIC calculations.

\subsection{Solar Model Results}

We calculated standard solar evolution models using the ATOMIC and OPAL opacities for the Asplund et al. (2009) (AGSS09) photospheric abundance mixture (see Guzik et al. (2015a, , b) )). The ATOMIC opacity tables were generated by mixing the pure-element OPLIB tables under the assumption of electron-temperature and electron-degeneracy equilibrium.

The solar models were calculated using an updated version of the Iben evolution code (see Guzik \& Mussack (2010) for details). The models include diffusive settling of helium and heavier elements relative to hydrogen. We adopt the usual symbols for hydrogen mass fraction (X), helium mass fraction $(\mathrm{Y})$, and mass fraction of all elements heavier than hydrogen and helium (Z), such that $\mathrm{X}+\mathrm{Y}+\mathrm{Z}=1$. The initial helium mass fraction and mixing length to pressure-scale-height ratio $(\alpha)$ are adjusted to calibrate the model to the observed solar luminosity $\left(3.846 \times 10^{33} \mathrm{erg} \mathrm{s}^{-1}\right)$ and radius $\left(6.9599 \times 10^{10} \mathrm{~cm}\right)$ at the present solar age $(4.54 \pm 0.04$ billion years). The initial $\mathrm{Z}$ is also adjusted so that, after diffusive settling, at the present solar age, the photospheric $\mathrm{Z} / \mathrm{X}=0.0181$, in agreement with the value derived by AGSS09. Table 1 summarizes the calibration parameters and other properties of the two solar models. The model evolved with the ATOMIC opacities has a slightly deeper convection zone than the model evolved with the OPAL opacities, but both models still show a too-shallow convection-zone depth and too-low convection zone helium mass fraction compared to the helioseismically inferred values from Basu \& Antia (2004).

Figure 4 shows helioseismically inferred (Basu et al. 2000) minus calculated sound speed vs. radius for the two models. The calculated sound speed profile is in better agreement with helioseismic inference using the ATOMIC opacities, although this change alone does not resolve the 


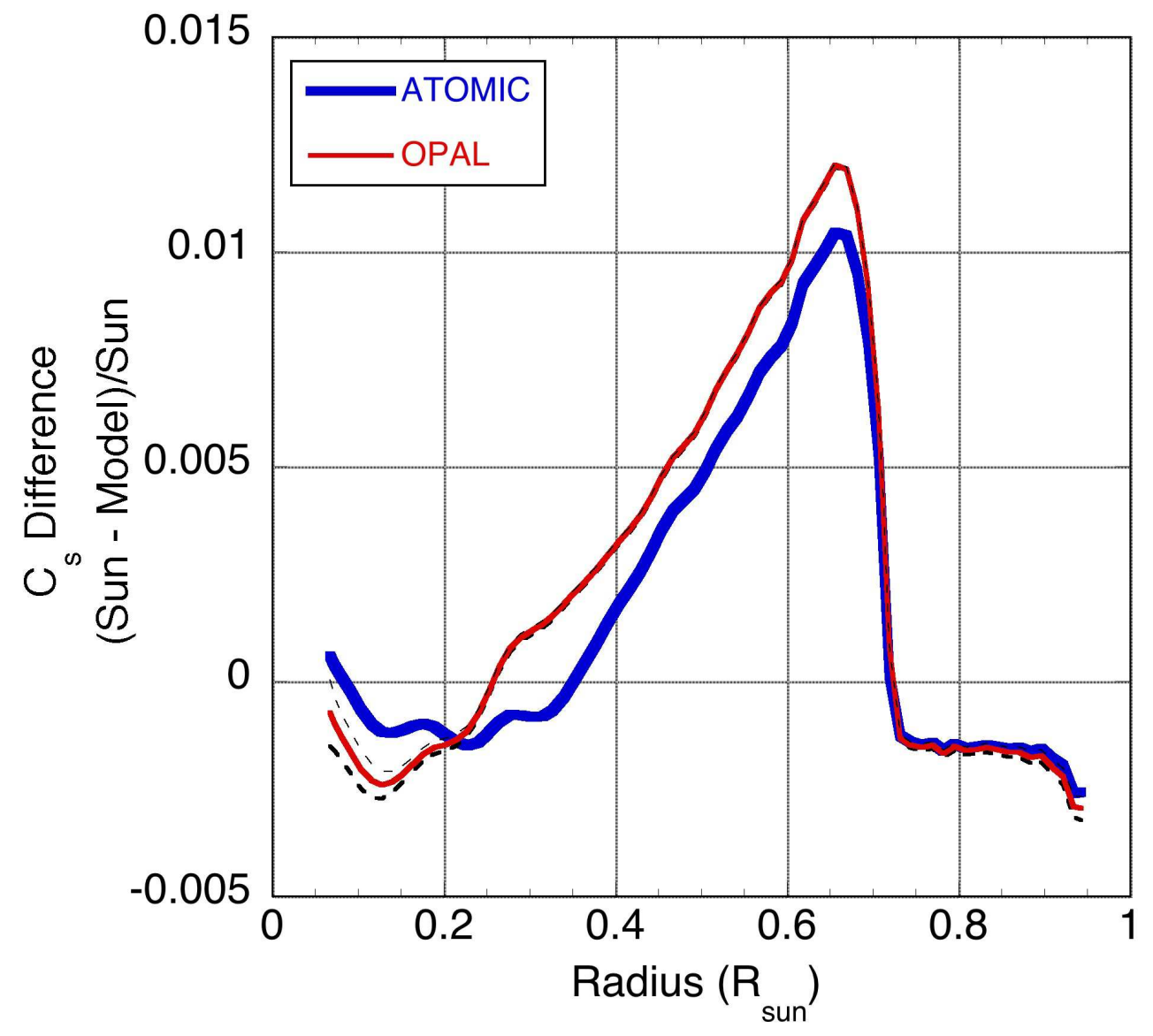

Fig. 4.- Helioseismically inferred (Basu et al. 2000) minus calculated sound speed differences vs. radius for solar models using the ATOMIC and OPAL opacities with the AGSS09 abundance mixture. The black dashed curves on either side of the OPAL profile show the magnitude of the uncertainty in the inferred sound-speed profile. The convection zone base radius is at $\sim 0.725 \mathrm{R}_{\odot}$. 


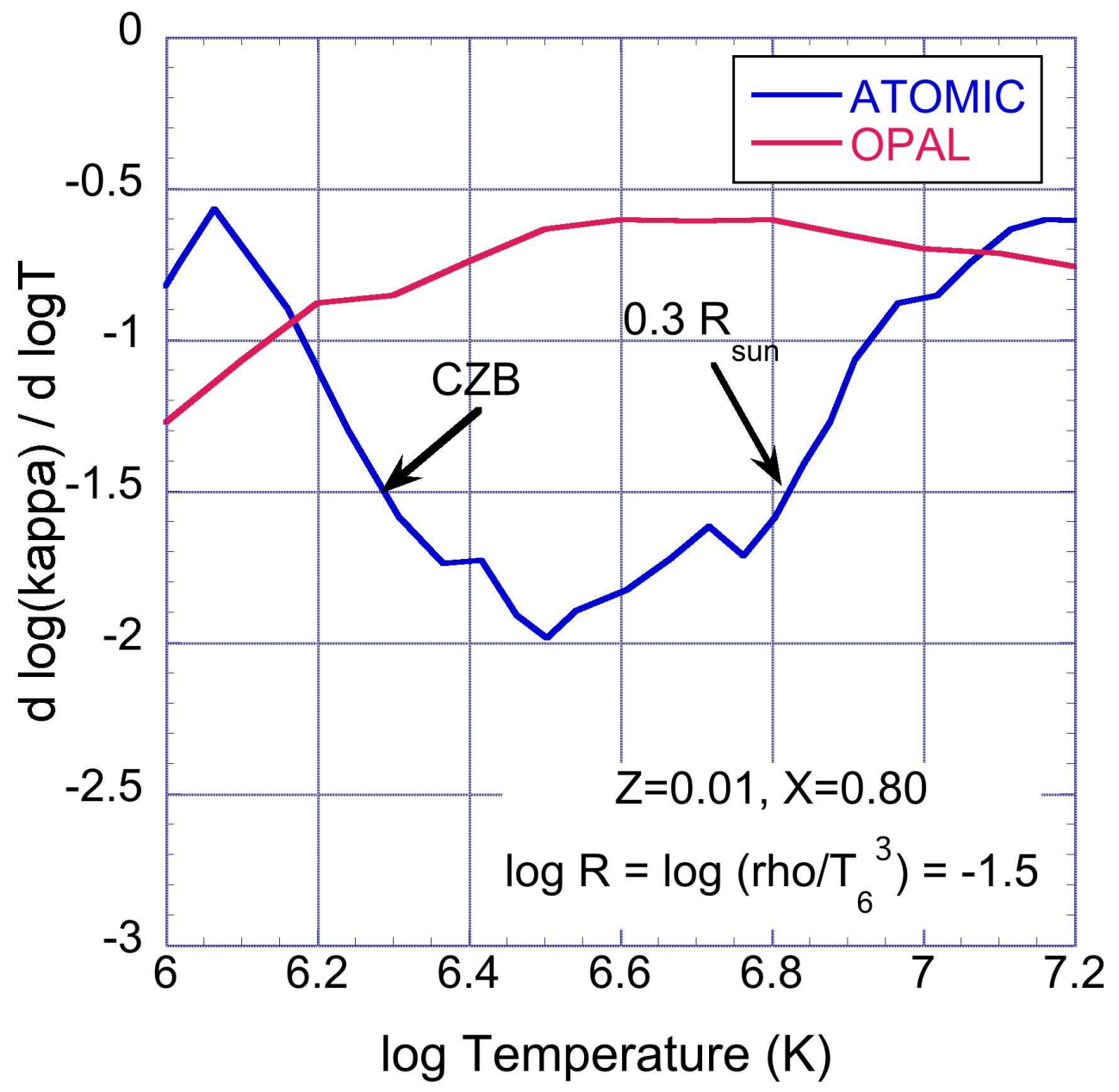

Fig. 5.- Logarithmic opacity derivative with respect to temperature for ATOMIC and OPAL tables with $\mathrm{Z}=0.01, \mathrm{X}=0.80$, and $\log R=-1.5$. The larger gradients for the ATOMIC table opacities at the convection-zone base $(\log T=6.3)$ and at $\sim 0.2-0.3 \mathrm{R}_{\odot}(\log \mathrm{T} \sim 6.8)$ in the solar interior are responsible for the differences in sound-speed gradients between models seen in Fig. 4 . 
discrepancy. We also found a similar improvement in agreement of sound speed profile with helioseismic inferences for solar models evolved using the AGSS09 abundance mixture calculated using the MESA code ((Paxton et al. 2015), see also Guzik et al. (2015a, b $)$ ).

To investigate the reason for the change in sound speed profile using the ATOMIC vs. the OPAL opacities, we compared the absolute values and the logarithmic temperature derivatives of the OPAL and ATOMIC opacities. We find that the OPAL opacities are actually slightly higher than the ATOMIC opacities for the entire solar radiative interior $(\log T=6.3$ to 7.2 ) but that the ATOMIC opacities become higher than OPAL in the solar convection zone, where opacity is not important to the solar structure because convection is transporting nearly all of the emergent luminosity. Figure 5 shows the logarithmic opacity derivatives with respect to temperature for the ATOMIC and OPAL table opacities for $\log R=-1.5, \mathrm{Z}=0.01$, and $\mathrm{X}=0.8$. The derivatives of the ATOMIC opacities are steeper than those of the OPAL opacities at the location of the solar convection zone base around $0.725 \mathrm{R}_{\odot}(\log T \sim 6.3)$, and near $\log T=6.8$, corresponding to $\sim 0.3$ $\mathrm{R}_{\odot}$. These are the locations that the sound-speed profile for the solar model using the ATOMIC opacities changes slope compared to the model for the OPAL opacities (Fig. 4).

\section{Conclusions}

In this paper we have discussed in detail the calculations of the new OPLIB opacity tables that have been generated using the Los Alamos ATOMIC code. Our calculations represent a systematic improvement in both the underlying physics approximations and in the amount of detail included in the calculations compared to the previous generation of Los Alamos opacity tables that were released around 15 years ago. A selection of our new monochromatic opacities have been discussed here and also in recent publications (Colgan et al. 2013a, b, 2015), where we presented detailed comparisons of ATOMIC and LEDCOP calculations. We have also demonstrated that use of our new opacity tables in solar modeling leads to improved agreement with helioseismology, although use of the new tables does not fully resolve the long-standing discrepancies. We also note that our new tables lead to improved agreement with observation of pulsations of B-stars (Walczak et al. 2015).

We hope that our new opacity tables, which are now publicly available2, will prove useful to the astrophysical modeling community. Further exploration of how these new opacities impact the understanding of a multitude of stellar objects is highly desirable and will be investigated in the near future.

The Los Alamos National Laboratory is operated by Los Alamos National Security, LLC for the National Nuclear Security Administration of the U.S. Department of Energy under Contract

\footnotetext{
${ }^{2}$ http://aphysics2.lanl.gov/opacity/lanl
} 
No. DE-AC52-06NA25396. We thank P. Walczak, who helped prepare the ATOMIC and OPAL tables for the solar model results presented here. We obtained LLNL opacities from the Lawrence Livermore National Laboratory OPAL Opacity Web site: http://opalopacity.llnl.gov/opal.html.

\section{Appendix: Rayleigh scattering details}

We approximate the low energy Rayleigh scattering contribution for all neutral atoms based on the static dipole polarizability of the neutral atom (Carson 1976). When more detailed treatments are available they are used in place of this approximation (currently for $\mathrm{H}, \mathrm{He}, \mathrm{Li}, \mathrm{C}, \mathrm{N}, \mathrm{O}$, $\mathrm{Ne}$, and Ar). The more detailed treatments are based on the dynamic (or frequency dependent) dipole polarizability (Tarafdar \& Vardya 1969). The following formulae have been implemented in ATOMIC for the scattering on the red wing of the first resonance line of the ground state of the neutral atom. Below we give expressions for the scattering transport cross section $\sigma$, where also $\hbar \omega$ is the photon energy (in eV), $E_{H}=13.6057 \mathrm{eV}$ is the Rydberg unit of energy and $\sigma_{T h}=8 \pi r_{0}^{2} / 3=$ $6.65246 \times 10^{-25} \mathrm{~cm}^{2}$ is the total Thomson scattering cross section.

Hydrogen:

From Lee (2005) we have

$$
\sigma / \sigma_{T h}=20.24\left(\frac{\hbar \omega}{2 E_{H}}\right)^{4}+239.2\left(\frac{\hbar \omega}{2 E_{H}}\right)^{6}+2256\left(\frac{\hbar \omega}{2 E_{H}}\right)^{8} .
$$

Helium:

From Dalgarno (1962); Dalgarno \& Kingston (1960), but rescaled so that the first term agrees with more accurate static dipole polarizability factors from Schwerdtfeger (2006), we have

$$
\sigma / \sigma_{T h}=1.913\left(\frac{\hbar \omega}{2 E_{H}}\right)^{4}+4.52\left(\frac{\hbar \omega}{2 E_{H}}\right)^{6}+7.90\left(\frac{\hbar \omega}{2 E_{H}}\right)^{8} .
$$

Lithium:

From Zeiss et al. (1977), using the expansion of the dynamic polarizability given by

$$
\alpha(\omega)=\frac{e^{2} \hbar^{2}}{m} \sum_{j=1}^{\infty}(\hbar \omega)^{2 j-2} S(-2 j)
$$

where the individual Cauchy moments are defined by

$$
S(-2 j)=\sum_{N} \frac{f_{N, I}}{\left(E_{N, I}\right)^{2 j}},
$$

we obtain

$$
\sigma / \sigma_{T h}=\left(\frac{\hbar \omega}{2 E_{H}}\right)^{4}\left(163.6+35038\left(\frac{\hbar \omega}{2 E_{H}}\right)^{2}+7.590 \times 10^{6}\left(\frac{\hbar \omega}{2 E_{H}}\right)^{4}\right)^{2}
$$


Carbon:

From Tarafdar \& Vardya (1969), but rescaled so that the first term agrees with more accurate static dipole polarizability factors from Schwerdtfeger (2006), we have

$$
\sigma / \sigma_{T h}=126.8\left(\frac{\hbar \omega}{2 E_{H}}\right)^{4}\left(1+12.87\left(\frac{\hbar \omega}{2 E_{H}}\right)^{2}+152.3\left(\frac{\hbar \omega}{2 E_{H}}\right)^{4}\right)
$$

Nitrogen:

From Tarafdar \& Vardya (1969), but rescaled so that the first term agrees with more accurate static dipole polarizability factors from Schwerdtfeger (2006), we have

$$
\sigma / \sigma_{T h}=54.91\left(\frac{\hbar \omega}{2 E_{H}}\right)^{4}\left(1+9.611\left(\frac{\hbar \omega}{2 E_{H}}\right)^{2}+78.03\left(\frac{\hbar \omega}{2 E_{H}}\right)^{4}\right)
$$

Oxygen:

From Tarafdar \& Vardya (1969), but rescaled so that the first term agrees with more accurate static dipole polarizability factors from Schwerdtfeger (2006), we have

$$
\sigma / \sigma_{T h}=36.63\left(\frac{\hbar \omega}{2 E_{H}}\right)^{4}\left(1+4.803\left(\frac{\hbar \omega}{2 E_{H}}\right)^{2}+23.44\left(\frac{\hbar \omega}{2 E_{H}}\right)^{4}\right)
$$

Neon:

From Dalgarno (1962); Dalgarno \& Kingston (1960), but rescaled so that the first term agrees with more accurate static dipole polarizability factors from Schwerdtfeger (2006), we have

$$
\sigma / \sigma_{T h}=7.129\left(\frac{\hbar \omega}{2 E_{H}}\right)^{4}\left(1+2.16\left(\frac{\hbar \omega}{2 E_{H}}\right)^{2}+4.92\left(\frac{\hbar \omega}{2 E_{H}}\right)^{4}\right) .
$$

Argon:

From Dalgarno (1962); Dalgarno \& Kingston (1960), but rescaled so that the first term agrees with more accurate static dipole polarizability factors from Schwerdtfeger (2006), we have

$$
\sigma / \sigma_{T h}=122.55\left(\frac{\hbar \omega}{2 E_{H}}\right)^{4}\left(1+2.48\left(\frac{\hbar \omega}{2 E_{H}}\right)^{2}+9.72\left(\frac{\hbar \omega}{2 E_{H}}\right)^{4}\right) .
$$

\section{Other Elements:}

When more complete expressions are not available, we use the static dipole polarizability approximation using $\alpha(\omega=0)$ to give the total cross section for scattering opacity as

$$
\sigma / \sigma_{T h}=\alpha(0)^{2}\left(\frac{\hbar \omega}{2 E_{H}}\right)^{4} .
$$

In the final expression given above, the dipole polarizability $\alpha(0)$ is in units of Bohr radii cubed. 
Finally, the scattering opacity contribution, $\kappa_{\mathrm{SCAT}}$, is given by

$$
\kappa_{\mathrm{SCAT}}=\frac{N \sigma}{\rho}
$$

with $\rho$ the mass density in $\mathrm{g} / \mathrm{cm}^{3}$, and $N$ the population of the ground state (in $\mathrm{cm}^{-3}$ ) of the neutral atom under consideration.

\section{REFERENCES}

Abdallah, Jr., J., Clark, R. E. H., \& Cowan, R. D. 1988, Los Alamos Manual LA-11436-M, Vol. I

Abdallah, Jr., J., Kilcrease, D. P., Magee, N. H., Mazevet, S., Hakel, P., \& Sherrill, M. E. 2007, High Energy Density Phys., 3, 309

Ali, A. W., \& Griem, H. R. 1965, Phys. Rev., 140, A1044

Ali, A. W., \& Griem, H. R. 1966, Phys. Rev., 144, 366

Armstrong, B. H., Johnson, R. R., DeWitt, H. E., \& Brush, S. G. 1966, Opacity of High Temperature Air, ed. C. A. Rouse (Progress in High Temperature Physics and Chemistry, Vol 1., Pergamon Press, Oxford, New York)

Armstrong, G. S. J., Colgan, J., Kilcrease, D. P., \& Magee Jr., N. H. 2014, High Energy Density Phys., 10, 61

Asplund, M., Grevesse, N., \& Sauval, A. J. 2005, in ASP Conf. Ser., 336, 25

Asplund, M., Grevesse, N., Sauval, A. J., \& Scott, P. 2009, ARA\&A, 47, 481

Badnell, N. R., Bautista, M. A., Butler, K., Delahaye, F., Mendoza, C., Palmeri, P., Zeippen, C. J., \& Seaton, M. J. 2005, MNRAS, 360, 458

Bahcall, J. N., Serenelli, A. M., \& Basu, S. 2005, ApJ, 621, L85

Bailey, J. E., Rochau, G. A., Iglesias, C. A., Abdallah Jr., J., MacFarlane, J. J., Golovkin, I., Wang, P., Mancini, R. C., Lake, P. W., Moore, T. C., Bump, M., Garcia, O., \& Mazevet, S. 2007, Phys. Rev. Letts., 99, 265002

Bailey, J. E., Nagayama, T., Loisel, G. P., Rochau, G. A., Blancard, C., Colgan, J., Cosse, Ph., Faussurier, G., Fontes, C. J., Gilleron, F., Golovkin, I., Hansen, S. B., Iglesias, C. A., Kilcrease, D. P., MacFarlane, J. J., Mancini, R. C., Orban, C., Pain, J.-C., Pradhan, A. K., Sherrill, M., \& Wilson, B. G. 2015, Nature, 517, 56

Basu, S. et al. 2000, ApJ, 529, 1084

Basu, S. \& Antia, H. M. 2004, ApJ, 606, L85 
Bell, K. L., Hibbert, A., \& Berrington, K. A. 1988, J. Phys. B, 21, 2319

Blancard, C., Cossé, P., \& Faussurier, G. 2012, ApJ, 745, 10

Carson, T. R. 1976, Ann. Rev. Ast. Ap., 14, 95

Chabrier, G. \& Potekhin, A. Y. 1998, Phys. Rev. E, 58, 4941

Clark, R. E. H., Abdallah Jr, J., \& Mann, J. B. 1991, ApJ, 381, 597; Abdallah Jr, J., Zhang, H. L., Fontes, C. J., Kilcrease, D. P., \& Archer, B. J. 2001, Journal of Quant. Spectrosc. Rad. Transfer, 71, 107

Colgan, J., Kilcrease, D. P., Magee, Jr., N. H., Armstrong, G. S. J., Abdallah, Jr., J., Sherrill, M. E., Fontes, C. J., Zhang, H. L., \& Hakel, P. 2013a, Proceedings of the International Conference on Atomic and Molecular Data, pp 17

Colgan, J., Kilcrease, D. P., Magee, Jr., N. H., Armstrong, G. S. J., Abdallah, Jr., J., Sherrill, M. E., Fontes, C. J., Zhang, H. L., \& Hakel, P. 2013b, High Energy Density Phys., 9, 369

Colgan, J., Kilcrease, D. P., Magee, Jr., N. H., Abdallah, Jr., J., Sherrill, M. E., Fontes, C. J., Zhang, H. L., \& Hakel, P. 2015, High Energy Density Phys., 14, 33

Cowan, R. D. 1981, The Theory of Atomic Structure and Spectra (University of California Press, Berkeley)

Cugier, H. 2012, A \& A, 42, 547

Dalgarno, A. 1962, Spectral Reflectivity of the Earth's Atmosphere III: The Scattering of Light by Atomic Systems, Geophysical Corporation of America Report No. 62-20-A

Dalgarno, A., \& Kingston, A. E. 1960, Proc. Royal Soc. London A, 259, 424

Däppen, W. 1987, ApJ, 319, 195

Daszyńska-Daszkiewicz, J., \& Walczak, P. 2009, MNRAS, 398, 1961

Daszyńska-Daszkiewicz, J., \& Walczak, P. 2010, MNRAS, 403, 496

Daszyńska-Daszkiewicz, J. Szewczuk, W., \& Walczak, P. 2013, MNRAS, 431, 3396

Davidson, S. J., Foster, J. M., Smith, C. C., Warburton, K. A., \& Rose, S. J. 1988, Appl. Phys. Letters, 52,847

Fontes, C. J., Zhang, H. L., Abdallah Jr, J., Clark, R. E. H., Kilcrease, D. P., Colgan, J., Cunningham, R. T., Hakel, P., Magee N. H., \& Sherrill, M. E. 2015, J. Phys. B, 48, 144014

Fontes, C. J., Colgan, J., \& Abdallah, Jr., J. 2016, Self-Consistent Large-Scale Collisional-Radiative Modeling, in Modern Methods in Collisional-Radiative Modeling of Plasmas, ed. by Yu. Ralchenko (Springer, New York), in press 
Geltman, S. 1962, ApJ, 136, 935

Geltman, S. 1965, ApJ, 141, 376

Gilles, D.,Turck-Chièze, S., Loisel, G., Piau, L., Ducret, J. E., Poirier, M., Blenski, T., Thais, F., Blancard, C., Cossé, P., Faussurier, G., Gilleron, F., Pain, J. C., Porcherot, Q., Guzik, J. A., Kilcrease, D. P., Magee, N. H., Harris, J., Busquet, M., Delahaye, F., Zeippen, C. J., \& Bastiani-Ceccotti, S. 2011, High Energy Density Phys., 7, 312

Green, J. M. 1958, RAND Corporation Report RM-2223-AEC

Green, J. M. 1960, RAND Corporation Report RM-2580-AEC

Grevesse, N., \& Noels, A. 1993, in Origin and Evolution of the Elements, 15

Grevesse, N. \& Sauval, A. J. 1998, Space Science Reviews 85, 161

Griem, H. R. 1974, Spectral Line Broadening by Plasmas (Academic Press, New York and London)

Guzik, J. A., \& Mussack, K. 2010, ApJ, 713, 1108

Guzik, J. A., Fontes, C. J., Walczak, P., Wood, S. R., Mussack, K., \& Farag, E. 2015a, Proc. International Astronomical Union Focus Meeting 17: Advances in Stellar Physics from Asteroseismology, Honolulu, HI, August 10-14, 2015, eds. C.S. Jeffery, J. A. Guzik, \& K. Pollard, Cambridge U. Press, in press.

Guzik, J. A., et al. 2015b, in preparation.

Hakel, P., \& Kilcrease, D. P. 2004, 14th Topical Conference on Atomic Processes in Plasmas, Eds: J. S. Cohen, S. Mazevet, and D. P. Kilcrease, (New York: AIP), pp 190

Hakel, P., Sherrill, M. E., Mazevet, S., Abdallah Jr., J., Colgan, J., Kilcrease, D. P., Magee, N. H., Fontes, C. J., \& Zhang, H. L. 2006, J. Quant. Spectrosc. Rad. Transfer, 99, 265

Heitler, W. 2010, The Quantum Theory of Radiation (3rd Edition, Dover Publications)

Hindmarsh, W. R., Petford, A. D., \& Smith, G. 1967, Proc. Roy. Soc. A, 297, 296

Hubeny, I., et al. 1994, A \& A, 282, 151

Huebner, W. F., \& Barfield, W. D. 2014, Opacity (Springer, New York)

Hummer, D., \& Mihalas, D. 1988, ApJ, 331, 794

Iglesias, C. 2010, High Energy Density Phys., 6, 311

Iglesias, C. 2015a, High Energy Density Phys., 15, 4

Iglesias, C. 2015b, MNRAS, 450, 2 
Iglesias, C. A., \& Rogers, F. J. 1996, ApJ, 464, 943

Kilcrease, D. P., Colgan, J., Hakel, P., Fontes, C. J., \& Sherrill, M. E. 2015, High Energy Density Phys., 16, 36

Kilcrease, D. P., \& Magee, N. H. 2001, J. Quant. Spectrosc. Radiat. Transfer, 71, 445

Lee, R. W. 1988, J. Quant. Spectrosc. Radiat. Transfer, 40, 561

Lee, H. W. 2005, MNRAS, 358, 1472

Le Pennec, M., Turck-Chièze, S., Salmon, S., Blancard, C., Cossé, P., Faussurier, G., \& Mondet, G. 2015, ApJ, 813, L42

Magee, N. H., Abdallah, Jr., J., Clark, R. E. H., Cohen, J. S., Collins, L. A., Csanak, G., Fontes, C. J., Gauger, A. Keady, J. J., Kilcrease, D. P., \& Merts, A. L. 1995, Astronomical Society of the Pacific Conference Series, 78, 51

Magee, N. H., Abdallah, J., Colgan, J., Hakel, P., Kilcrease, D. P., Mazevet, S., Sherrill, M., Fontes, C. J., \& Zhang, H. L. 2004, 14th Topical Conference on Atomic Processes in Plasmas, Eds: J. S. Cohen, S. Mazevet, and D. P. Kilcrease, (New York: AIP), pp 168

Mazevet S., \& Abdallah, Jr., J. 2006, J. Phys. B, 39, 3419

Mondet, G., Blancard, C., Cossé, P., \& Faussurier, G. 2015, ApJ SS, 220, 2

Nagayama, T., Bailey, J. E., Loisel, G., Hansen, S. B., Rochau, G. A., Mancini, R. C., MacFarlane, J. J., \& Golovkin, I. 2014, Phys. Plasmas, 21, 056502

Nakagawa, M., Kohyama, Y., \& Itoh, N. 1987, ApJ SS, 63, 661

Neuforge-Verheecke, C., Guzik, J. A., Keady, J. J., Magee, N. H., Bradley, P. A., \& Noels, A. 2001, ApJ, 561, 450

Neuforge, C., Goriely, S. Guzik, J. A., Bradley, P. A., \& Swenson, F. J. 2001, ApJ, 550, 493

Pain, J.-C., \& Gilleron, F. 2015, High Energy Density Phys., 15, 30

Paxton, B. et al., 2015, ApJ, 220, 15

Perry, T. S., Davidson, S. J., Serduke, F. J., Bach, D. R., Smith, C. C., Foster, J. M., Doyas, R. J., Ward, R. A., Iglesias, C. A., Rogers, F. J., Abdallah, Jr., J., Stewart, R. E., Kilkenny, J. D., \& Lee, R. W. 1991, Phys. Rev. Letts., 67, 3784

Potekhin, A. Y., \& Chabrier, G. 2000, Phys. Rev. E, 62, 8554

Potekhin, A. Y., Chabrier, G., \& Gilles, D. 2002, Phys. Rev. E, 65, 036412

Rogers, F. J., \& Iglesias, C. A. 1992, ApJS, 79, 507 
Sampson, D. H. 1959, ApJ, 129, 734

Schwerdtfeger, X. 2006, Atomic Static Dipole Polarizabilities, in Computational Aspects of Electric Polarizability Calculations, (IOS Press) \& http://ctcp.massey.ac.nz/Tablepol-2.8.pdf

Seaton, M. J., Yan, Y., Mihalas, D., \& Pradhan, A. K. 1994, MNRAS, 266, 805

Seaton, M. J., \& Badnell, N. R. 2004, MNRAS, 354, 457

Serenelli, A. M., Basu, S., Ferguson, J. W., \& Asplund, M. 2009, ApJ, 705, L123

Somerville, W. B. 1965, ApJ, 141, 811

Springer, P. T., Fields, D. J., Wilson, B. G., Nash, J. K., Goldstein, W. H., Iglesias, C. A., Rogers, F. J., Swenson, J. K., Chen, M. H., Bar-Shalom, A., \& Stewart, R. E. 1992, Phys. Rev. Letts., 69, 3735

Tarafdar, S. P., \& Vardya, M. S. 1969, MNRAS, 145, 171

Turck-Chièze, S., Gilles, D., Le Pennec, M., Blenski, T., Thais, F., Bastiani-Ceccotti, S., Blancard, C., Busquet, M., Caillaud, T., Colgan, J., Cossè, P., Delahaye, F., Ducret, J. E., Faussurier, G., Fontes, C. J., Gilleron, F., Guzik, J., Harris, J. W., Kilcrease, D. P., Loisel, G., Magee, N. H., Pain, J. C., Reverdin, C., Silvert, V., Villette, B., \& Zeippen, C. J. 2013, High Energy Density Phys., 9, 473

Walczak, P., Fontes, C. J., Colgan, J., Kilcrease, D. P., \& Guzik, J. A. 2015, A\&A, 580, L9

Weiss, A., Hillebrandt, W., Thomas, H.-C., \& Ritter, H. 2004, Cox \& Giuli's principles of stellar structure (Extended Second Edition, Cambridge Scientific Publishing)

Winhart, G., Eidmann, K., Iglesias, C. A., \& Bar-Shalom, A. 1996, Phys. Rev. E, 53, R1332

Zeiss, G. D. \& Meath, W. J. 1977, Mol. Phys., 33, 1155 
Table 1: Calibration parameters and properties of solar models evolved using OPAL or ATOMIC opacities for the AGSS09 abundance mixture.

\begin{tabular}{lcc}
\hline & OPAL & ATOMIC \\
\hline $\mathbf{Y}_{\text {initial }}$ & 0.2641 & 0.2570 \\
$\mathbf{Z}_{\text {initial }}$ & 0.0150 & 0.0151 \\
$\alpha$ & 2.0118 & 2.0637 \\
$\mathbf{Y}_{\text {conv.zone }}{ }^{a}$ & & \\
$\mathbf{Z}_{\text {conv.zone }}{ }_{\mathbf{R}_{\text {conv.zone base }}{ }^{b}}\left(\mathbf{R}_{\odot}\right)$ & 0.2345 & 0.2283 \\
\hline
\end{tabular}

${ }^{a}$ Helioseismically inferred convection-zone Y is $0.248 \pm 0.003$ (Basu \& Antia 2004)

${ }^{b}$ Helioseismically inferred convection-zone radius is $0.713 \pm 0.001 \mathrm{R}_{\odot}$ (Basu \& Antia 2004) 


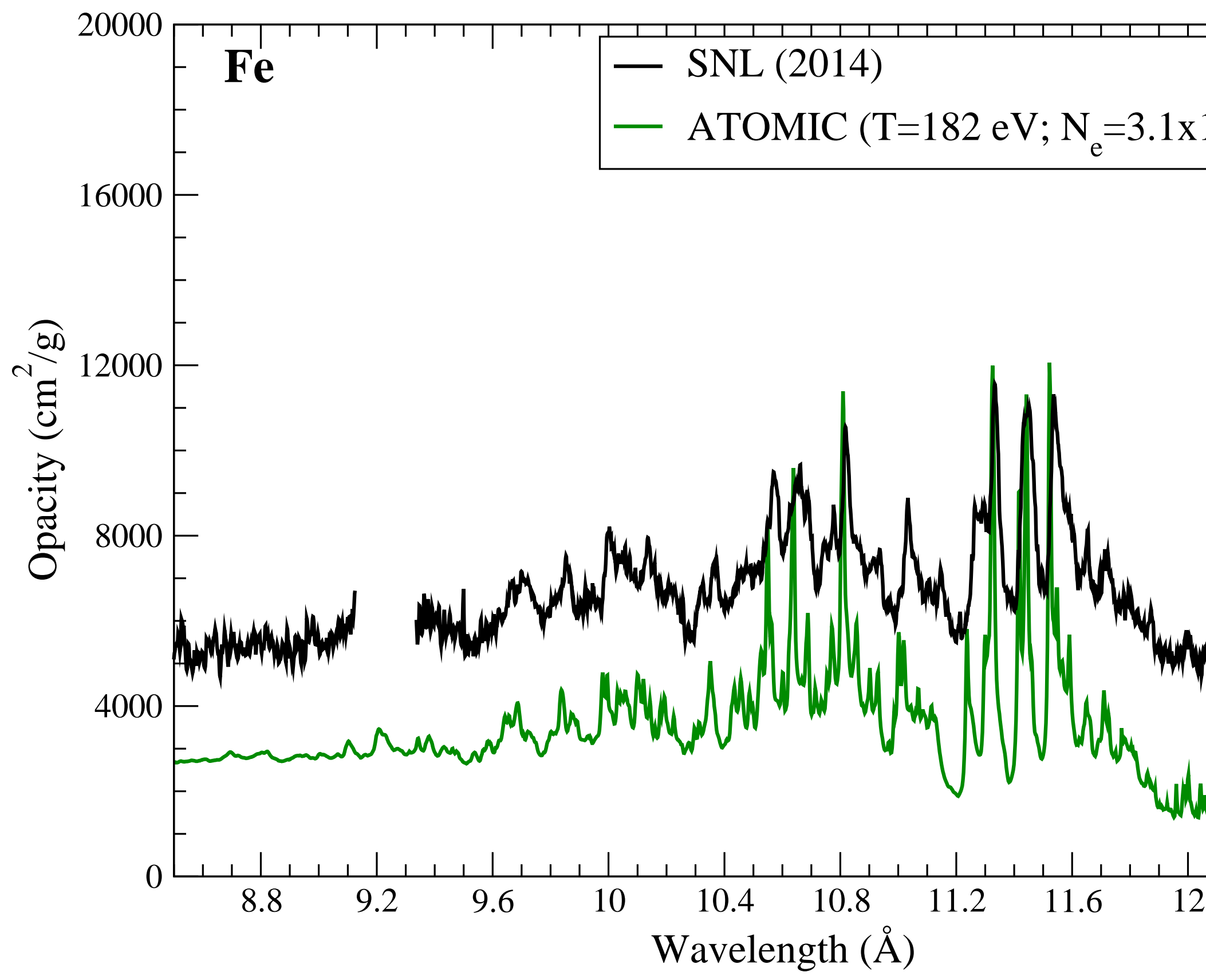

